\title{
Effect of Pueraria mirifica on the Sexual Skin Coloration of Aged Menopausal Cynomolgus Monkeys
}

\author{
Hataitip TRISOMBOON ${ }^{1,2)}$, Suchinda MALAIVIJITNOND ${ }^{2)}$, \\ Wichai CHERDSHEWASART ${ }^{2)}$, Gen WATANABE ${ }^{3,4)}$ and Kazuyoshi TAYA ${ }^{3,4)}$ \\ 1) Department of Physiology, Faculty of Medicine, Srinakharinwirot University, Bangkok \\ 10110, ${ }^{2}$ Primate Research Unit, Department of Biology, Faculty of Science, Chulalongkorn \\ University, Bangkok 10330, Thailand, ${ }^{3)}$ Laboratory of Veterinary Physiology, Department of \\ Veterinary Medicine, Faculty of Agriculture, Tokyo University of Agriculture and Technology, \\ Fuchu, Tokyo 183-8509 and ${ }^{4)}$ Department of Basic Veterinary Science, The United Graduate \\ School of Veterinary Science, Gifu University, Gifu 501-1193, Japan
}

\begin{abstract}
To investigate the estrogenic effect of Pueraria mirifica (PM), a Thai herbal plant that contains many phytoestrogens, sexual skin coloration was studied in cynomolgus monkeys. Aged menopausal monkeys were divided into three groups. Each group $(n=3)$ was fed 10, 100, or 1,000 mg of PM daily. The treatment schedule was divided into three periods, a 30-day pre-treatment period, 90-day treatment period, and 60-day post-treatment period. The results show that the sexual skin exhibited reddish coloration within $24 \mathrm{~h}$ after PM-treatment and remained this way for the first half of the PMfeeding period. The changes in sexual skin coloration were not dose-dependent. The present results indicate that PM had estrogenic action by increasing reddish sexual skin coloration in aged menopausal monkeys.
\end{abstract}

Key words: Macaca fascicularis, Phytoestrogens, Pueraria mirifica, Sexual skin

(J. Reprod. Dev. 52: 537-542, 2006)

$\mathrm{t}$ has been recognized that the roots of Pueraria mirifica (PM), a Thai herbal plant, contain many kinds of phytoestrogens, such as genistein, daidzein, and coumestrol, which are also found in soy plants and soy products, and miroestrol, deoxymiroestrol, peurarin, and mirificin [1-4]. The phytoestrogens from soys [5, 6] and PM roots [7-9] have been reported to have estrogenic actions in both in vivo and in vitro studies. The estrogenic effect of PM was examined in vitro using MCF-7 cell proliferation and HepG2 cell transient transfection assay $[7,8]$. PM had a dual effect on the growth of

Accepted for publication: April 21, 2006

Published online: June 24, 2006

Correspondence: S. Malaivijitnond

(e-mail: Suchinda.M@chula.ac.th)
MCF-7 human mammary adenocarcinoma cells after 4 days of incubation, i.e. stimulating growth at a low dose and suppressing it with a high dose [8]. PM also showed estrogenic activity on a human hepatoma cell line, HepG2 cells, containing estrogen receptor and luciferase reporter gene [7]. Administration of PM produced cornified cells, growth of the endometrium gland, and proliferation of spiral arteries in ovariectomized rats [9]. Furthermore, we reported in a recent investigation that daily feeding of PM had an estrogenic effect by suppressing gonadotropin and estrogen secretions in aged female cynomolgus monkeys [10].

The influence of estrogen on the sexual skin area around the perineum to the base of the tail has been 
reported for the nonhuman old world primates [11, 12]. The color of the skin of rhesus monkeys (Macaca mulatta) has been shown to be correlated with high concentrations of estrogen before ovulation, whereas the skin shows its palest coloration at menstruation $[13,14]$. In addition, the sexual skin of cynomolgus monkeys (Macaca fascicularis) [15], pig-tailed macaques (Macaca nemestrina) [16], and mangabey monkeys (Cercocebus atys lunulatus) [11] display maximal edema and swelling associated with estrogen fluctuation during the menstrual cycle. Administration of estrogen caused increasing redness of the sexual skin area of ovariectomized rhesus monkeys $[13,16]$ and intact male rhesus monkeys [12]. The sexual skin is thus considered to be an external indicator of estrogenic effect in monkeys. The coloration of the sexual skin of female cynomolgus monkeys has been related to estrogenic level and has been observed more clearly than sexual swelling as an indicator of estrogenic action. The aim of the present study was, therefore, to investigate the estrogenic effect of PM on the changes in sexual skin of aged female cynomolgus monkeys.

\section{Materials and Methods}

\section{Animals}

Nine aged menopausal cynomolgus monkeys (Macaca fascicularis) over 20 years of age and weighing 4.0 to $6.0 \mathrm{~kg}$ were selected from the colony of the Primate Research Unit, Chulalongkorn University. All monkeys had completed cessation of menstrual cyclicity at least one year before the start of this study. The monkeys were monitored for menstruation and vaginal discharge daily by the vaginal swabbing method before and throughout the experiment. In addition, monkey health and behavior were also observed throughout the study period. Monkeys were housed in individual cages, fed a monkey feed daily (Pokphand Animal Feed Co., Ltd., Bangkok, Thailand) in the morning (0900 to $1000 \mathrm{~h}$ ), and given fresh fruit in the afternoon (1400 to 1500 $\mathrm{h})$. The lighting conditions of the animal house were controlled with a $12 \mathrm{~h}$ light/12 h dark cycle. Temperature and humidity fluctuated slightly depending on the season. The experimental protocol was approved in accordance with the guide for care and use of laboratory animals prepared by Chulalongkorn University.

\section{Protocol of the study}

The nine monkeys were randomly divided into three groups. Each monkey group $(n=3)$ was fed daily a suspension of 10,100, or 1,000 mg of PM in 5 $\mathrm{ml}$ of distilled water per monkey, henceforth referred to as PM-10, PM-100, and PM-1,000, respectively. The experiment term was divided into 30-day pre-treatment, 90-day treatment, and 60-day post-treatment periods. Each monkey was fed 5-ml distilled water daily during the pre- and post-treatment periods and PM-10, PM-100, or PM1,000 during the treatment period between 0800 to $0830 \mathrm{~h}$. A change in sexual skin color was observed daily between 0830 to $0900 \mathrm{~h}$ throughout the study. The coloration of the sexual skin of cynomolgus macaques varies between individuals as shown in the pre-treatment period in Fig. 1. Therefore, to validate the results, the changes in sexual skin color of each monkey after the PM treatment were compared with their own sexual skin color during the pre-treatment period.

\section{Preparation of the PM suspension}

Fresh tuberous PM roots from Cultivar-Wichai III collected in Chiang Mai, Thailand, were sliced, desiccated in a hot air oven at $70 \mathrm{C}$, and subsequently ground into powder. The PM powder was kept in dark desiccators before preparation of the PM suspension. The dried PM root powder was suspended in $5 \mathrm{ml}$ of distilled water and kept in a dark bottle at $4 \mathrm{C}$ prior to feeding.

\section{Investigation of sexual skin coloration}

Visual observation of the coloration of the sexual skin, the area around the perineal area to the base of the tail, was conducted by comparing with a reference marker. The skin coloration was graded as 1 (palest), 2 (red), or 3 (dark red), as shown in Fig. 1.

\section{Statistical analysis}

The changes in sexual coloration of monkeys during the PM-treatment and post-treatment periods compared with the pre-treatment periods were evaluated by Wilcoxon two-related sample test. The criterion for significance was set at $P<0.05$. 

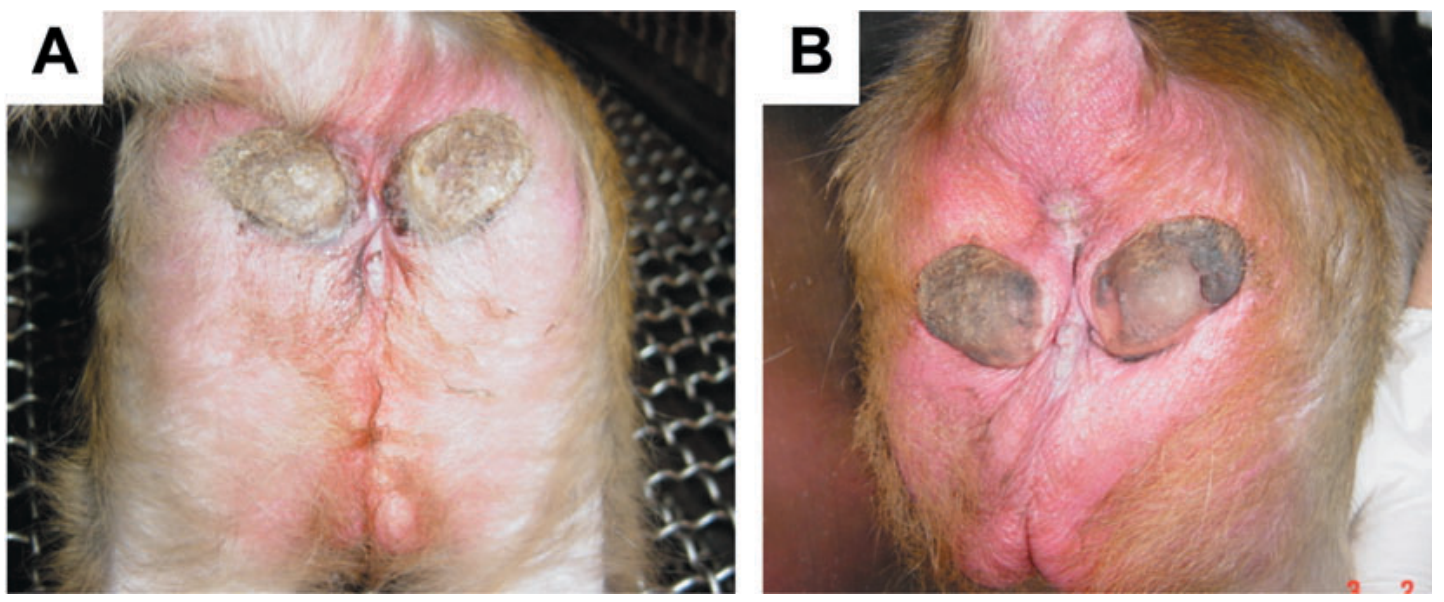

Fig. 1. Sexual skin coloration of monkeys showed their palest color, grade 1 (panel A), during the pre-treatment period and a reddish color, grade 3 (panel B), within $24 \mathrm{~h}$ of treatment with PM-10, PM-100, or PM-1,000.

\section{PM-10}
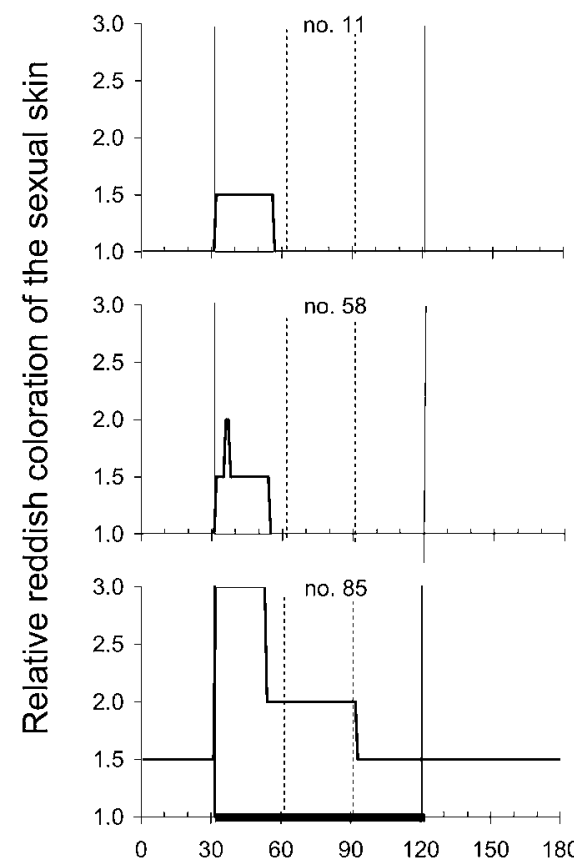

PM-100
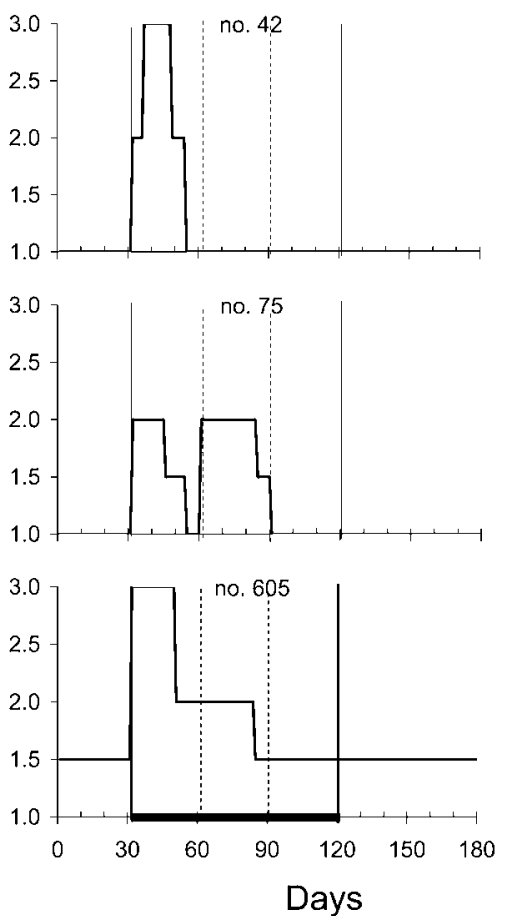

PM-1,000
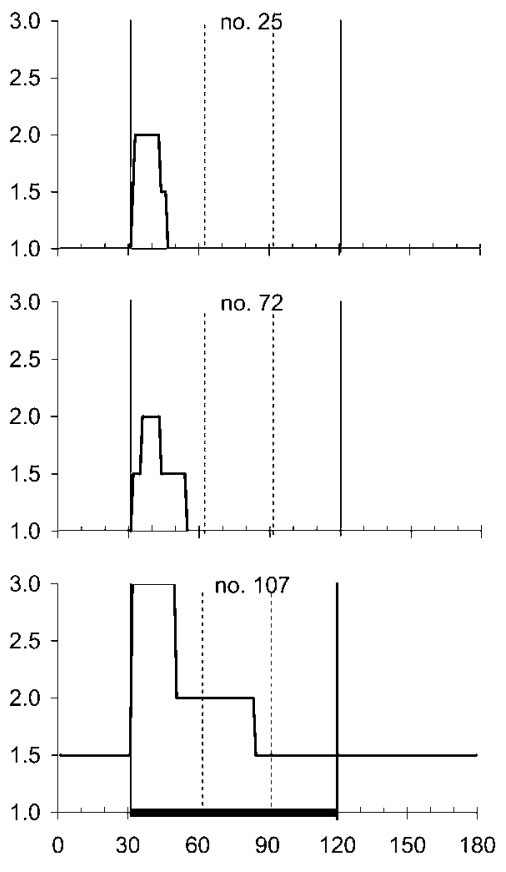

Fig. 2. The coloration of the sexual skin of each aged menopausal monkey treated with PM-10, PM-100, or PM-1,000. The horizontal bars show the 90-day PM-feeding period. The dashed vertical lines show each 30 -day period of the treatment period.

\section{Results}

Menstruation and vaginal discharge were not observed throughout the study period. Moreover, no changes in monkey activity were detected. As shown in Fig. 2, although we could not find any statistically significant differences in sexual skin coloration between the pre-treatment, treatment, and post-treatment periods, the aged menopausal monkeys had their palest sexual skin coloration, 
graded between 1 and 1.5, throughout the pretreatment period. After PM feeding, a reddish coloration of the sexual skin was observed within $24 \mathrm{~h}$ for all the PM-treated groups. In PM-10, the reddish sexual skin coloration was observed for 24 to 26 days in two monkeys and for 62 days in the other monkey. Likewise, the sexual skin of one monkey treated with PM-100 exhibited a reddish coloration for 24 days and the other two exhibited a reddish coloration for 54 to 60 days. Two monkeys treated with PM-1,000 exhibited a reddish coloration for 16 to 24 days and the other monkey exhibited a reddish coloration for 54 days. Although some monkeys in all the dose groups showed a longer period of reddish coloration, grade 2 (62 days in the PM-10 group, 54 to 60 days in the PM-100 group, and 54 days in the PM-1,000 group), a darker reddish coloration, grade 3 , was only exhibited for 2 to 21 days in the PM-10 group, 11 to 17 days in the PM-100 group, and 7 to 19 days in the PM-1,000 group. Therefore, the changes in sexual skin color varied in each monkey and did not depend on the PM dose.

\section{Discussion}

In the present study, daily feeding of PM containing phytoestrogens produced an estrogenic effect on the sexual skin by increasing its reddish coloration and pigmentation, but not in a dosedependent manner. Increased redness of the sexual skin has long been considered an external indicator of estrogenic effect in nonhuman primates [11, 13]. Administration of estrogen and testosterone for 4 days induced an increasing reddish coloration of the sexual skin in intact male rhesus monkeys, while there was no effect in monkeys treated with either dihydrotestosterone or fadrozole, an aromatase inhibitor [12]. These results indicate that estrogen had a direct effect on the sexual skin, while testosterone had an indirect effect by acting through aromatization [12]. The effect of estrogen administration on the reddish coloration and swelling of the skin has also been similarly found in both intact and ovariectomized rhesus monkeys [14, 18].

Pigmentation of the skin is modulated by estrogen. Estrogen modulates cell proliferation and melanogenesis of normal human skin cultures [1921]. Estrogen-treated ovariectomized guinea pigs increased production of melanin and produced increased skin pigmentation [22, 23]. Furthermore, PM administration induced the proliferation of human mammary adenocarcinoma cells in vitro [8] and proliferation of the endometrium and spiral arteries in ovariectomized rats [9].

Previous studies have shown cytosol and nuclear estrogen receptors (ERs) in the sexual skin of Japanese monkeys (Macaca fuscata fuscata) [24]. Daily injection of mestranol, a synthetic estrogen, induced an increase in both cytosol and nuclear ERs positively correlated with sexual skin swelling. Furthermore, the nuclear ERs increased approximately by 4 -fold from the early to late follicular phases [24]. Hence, from these evidences, estrogen may influence the sexual skin via ERs in the sexual skin and produce the reddish coloration.

Although phytoestrogens are the nonsteroidal compound, their phenolic structures are closely related to those of estrogens, and the distances between the hydroxyl groups are also identical [25]. Therefore, based on structural similarities, phytoestrogens can exhibit estrogenic effect by binding both ER $\alpha$ and ER $\beta$ [26]. Genistein and daidzein showed agonist activity on rat ER $\alpha$ and $\mathrm{ER} \beta$ in vitro and were more potently bound to $\mathrm{ER} \beta$ than ER $\alpha$ [27-29], while both ERs have been found in animal and human skins, especially $\operatorname{ER} \beta$ [23]. Thus, it can be presumed in the present study that PM phytoestrogens bound to ERs and reddened the sexual skin.

Moreover, the menopausal monkeys had a highly sensitive reddish coloration response to all PM doses $(10,100$, and 1,000 mg/day) by producing a reddish coloration within $24 \mathrm{~h}$. Nevertheless, the increasing redness of the sexual skin in the monkeys returned to the palest color in the second half of the PM-treatment period. The decreasing redness of the sexual skin may be due to the high concentration of phytoestrogens in the circulation leading to an apparent down-regulation of ERs and reduced responsiveness. A high dose of phytoestrogen genistein induced down-regulation of ER $\alpha$ mRNA and protein levels in human breast cancer cell lines [29]. Changes in ER seem to be very rapid, since down-regulation of ER occurred within 6-12 h in endometrium cells of prepubertal lambs treated with estrogen [30]. This report supports our presumption that PM phytoestrogens may bind and modulate ER in sexual skin.

However, the change in sexual skin coloration 
was not concomitant with the change in endogenous estradiol level in serum [10]. Serum estradiol levels were unchanged, but tended to decrease in three monkey groups in a previous study [10]. This evidence suggests that the appearance of sexual color changes do not result from endogenous estrogen and are probably a direct influence from PM phytoestrogens. In summary, PM containing phytoestrogens may have an estrogenic effect on sexual skin coloration in menopausal cynomolgus monkeys.

\section{Acknowledgements}

This work was supported by The Thailand Research Fund (TRF) contract no. PHD/0014/2544 for the Royal Golden Jubilee Program (RGJ network) and no. BGJ/15/2544, Chulalongkorn University, Thailand; a Grant-in-Aid for Scientific Research (21 ${ }^{\text {st }}$ Century Center-of-Excellence Program, E-1) from the Ministry of Education, Culture, Sports, Science and Technology of Japan; and a Grant-in-Aid for Scientific Research (No.05480) from the Japan Society for the Promotion of Science.

\section{References}

1. Pope GS, Grundy HM, Jones HEH, Tait SAS. The estrogenic substance (miroestrol) from the tuberous roots of Pueraria mirifica. J Endocrinol 1958; 17: 15-16.

2. Ingham JL, Tahara $\mathbf{S}$, Dziedzic SZ. Minor isoflavones from the roots of Pueraria mirifica. Z Naturforsch 1988; 44c: 724-726.

3. Ingham JL, Tahara S, Dziedzic SZ. Coumestans from the roots of Pueraria mirifica. Z Naturforsch 1989; 43c: 5-10.

4. Chansakaow S, Ishikawa T, Seki H, Sekine K, Okada M, Chaichantipyuth C. Identification of deoxymiroestrol as the actual rejuvenating principle of "Kwao Keur" Pueraria mirifica. The known miroestrol may be an artifact. J Nat Prod 2000; 63: 173-175.

5. Diel P, Geis RB, Caldarelli A, Schmidt S, Leschowsky UL, Voss A, Vollmer G. The differential ability of the phytoestrogen genistein and of estradiol to induce uterine weight and proliferation in the rat in associated with a substance specific modulation of uterine gene expression. Mol Cell Endocrinol 2004; 221: 21-32.

6. Duncan AM, Berz BE, Xu X, Nagel T, Phipps W, Kurzer MS. Soy isoflavones exert modest hormonal effects in premenopausal women. J Clin Endocrinol Metab 1999; 84: 192-197.

7. Lee YS, Park LS, Cho SD, Son JK, Cherdshewasart W, Kang KS. Requirement of metabolic activation for estrogenic activity of Pueraria mirifica. J Vet Sci 2002; 3: 273-277.

8. Cherdshewasart $\mathbf{W}$, Cheewasopit $\mathbf{W}$, Picha $\mathbf{P}$. The differential anti-proliferation effect of white (Pueraria mirifica), red (Butea superba), and black (Mucuna collettii) kwao krua plants on the growth of MCF-7 cells. J Ethnopharmacol 2004; 93: 255-260.

9. Sukavattana T. Oestrogenic principle of Butea superba, preliminary report. J Med Assoc Thai 1940; 24: 183-194.
10. Trisomboon $\mathbf{H}$, Malaivijitnond S, Watanabe G, Taya K. The estrogenic effect of Pueraria mirifica on gonadotrophin levels in aged monkeys. Endocrine 2006; 29: 129-134.

11. Aidara D, Badawi M, Tahiri-Zagret C, Robyn C. Changes in concentrations of serum prolactin, $\mathrm{FSH}$, oestradiol and progesterone and of the sex skin during the menstrual cycle in the mangabey monkey. J Reprod Fert 1981; 62: 475-481.

12. Rhodes L, Argersinger ME, Gantert LT, Friscino BH, Hom G, Pikounis B, Hess DL, Rhodes WL. Effects of administration of testosterone, dihydrotestosterone, oestrogen and fadrozole, an aromatase inhibitor, on sex skin colour in intact male rhesus macaques. J Reprod Fert 1997; 111: 5157.

13. Czaja JA, Robinson JA, Eisele SG, Scheffler G, Goy RW. Relationship between sexual skin colour of female rhesus monkeys and midcycle plasma levels of oestradiol and progesterone. J Reprod Fert 1977; 49: 147-150.

14. Ruiz de Elvira MC, Herndon JG, Collins DC. Effect of estradiol-treated females on all-female groups of rhesus monkeys during the transition between the nonbreeding and breeding seasons. Folia Primatol 1983; 41: 191-203.

15. Englhardt A, Hodges JK, Niemitz C, Heistermann M. Female sexual behavior, but not sex skin swelling, reliably indicates the timing of the fertile phase in wild long-tailed macaques (Macaca fascicularis). Horm Behav 2005; 47: 195-204.

16. Carlisle KS, Brenner RM, Montagna W. Hormonal regulation of sex skin in Macaca nemestrina. Biol Reprod 1981; 25: 1053-1063.

17. Michael RP, Saayman GS. Differential effects on behaviour of the subcutaneous and intravaginal administration of oestrogen in the rhesus monkeys [Macaca mulatta]. J Endocrinol 1968; 41: 231-246. 
18. Golub MS, Hogrefe CE, Germann SL, Lasley BL, Natarajan K, Tarantal AF. Effects of exogenous estrogenic agents on pubertal growth and reproductive system maturation in female rhesus monkeys. Toxicol Sci 2003; 74: 103-113.

19. Jee SH, Lee SY, Chiu HC, Chang CC, Chen TJ. Effect of estrogen and estrogen receptor in normal human melanocytes. Biochem Biophys Res Commun 1994; 199: 1407-1412.

20. McLeod SD, Ranson M, Mason RS. Effects of estrogens on human melanocytes in vitro. J Steroid Biochem Mol Biol 1994; 49: 9-14.

21. Slominski A, Wortsman J. Neuroendocrinology of the skin. Endocr Rev 2000; 21: 457-487.

22. Snell RS, Bischitz PG. The Effect of large doses of estrogen and progesterone on melanin pigmentation. J Invest Dermatol 1960; 35: 73-82.

23. Thornton MJ. The biological actions of estrogens on skin. Exp Dermatol 2002; 11: 487-502.

24. Onouchi T, Kato J. Estrogen receptors and estrogen-inducible progestin receptors in the sexual skin of the monkey. J Steroid Biochem 1983; 18: 145151.

25. Setchell KD. Phytoestrogens: the biochemistry, physiology, and implications for human health of soy isoflavones. Am J Clin Nutr 1998; 68 (Suppl): 1333s-1346s.
26. Santell RC, Chang YC, Nair MG, Helferich WG. Dietary genistein exerts estrogenic effects upon the uterus, mammary gland, and the hypothalamic/ pituitary axis in rats. J Nutr 1997; 127: 263-269.

27. Kuiper, GGJM, Carlsson B, Grandien K, Enmark E, Haggblad J, Nilsson S, Gustafsson JA. Comparison of the ligand binding specificity and transcript tissue distribution of estrogen receptors $\alpha$ and $\beta$. Endocrinology 1997; 138: 863-870.

28. Casanova M, You L, Gaido KW, Archibeque-Engle S, Janszen DB, Heck H. Developmental effects of dietary phytoestrogens in spraque-dawley rats and interactions of genistein and daidzein with rat estrogen receptor $\alpha$ and $\beta$ in vitro. Toxicol Sci 1999. 51: 236-244.

29. Maggiolini M, Bonofiglio D, Marsico S, Panno ML, Cenni B, Picard D, Ando S. Estrogen receptor $\alpha$ mediates the proliferative but not the cytotoxic dose-dependent effects of two major phytoestrogens on human breast cancer cells. Mol Pharmacol 2001; 60: 595-602.

30. Meikle A, Bielli A, Masironi B, Penrana G, Wang H, Forsberg M, Sahlin L. An immunohistochemical study on the regulation of estrogen receptor $\alpha$ by estradiol in the endometrium of the immature ewe. Reprod Nutr Dev 2000; 40: 587-596. 